\title{
ON DECOMPOSITIONS OF MATRICES INTO PRODUCTS OF COMMUTATORS OF INVOLUTIONS*
}

\author{
TRAN NAM SON ${ }^{\dagger \ddagger}$, TRUONG HUU DUNG ${ }^{\S}$, NGUYEN THI THAI HA ${ }^{\dagger \ddagger}$, AND MAI HOANG BIEN ${ }^{\dagger \ddagger}$
}

\begin{abstract}
Let $F$ be a field and let $n$ be a natural number greater than 1 . The aim of this paper is to prove that if $F$ contains at least three elements, then every matrix in the special linear group $\operatorname{SL}_{n}(F)$ is a product of at most two commutators of involutions.
\end{abstract}

Key words. Matrix decomposition, Commutator, Involution, Special linear group.

AMS subject classifications. 15A23, 20H20.

1. Introduction and main results. The decomposition of a matrix into products of matrices having certain properties is an interesting topic that it has applications in many areas. In this paper, we focus on decompositions of matrices in special linear groups into products of commutators of involutions.

Let $F$ be a field, let $n$ be a natural number greater than 1, and let $\mathrm{GL}_{n}(F)$ be the general linear group of degree $n$ over $F$. Denote by $\mathrm{SL}_{n}(F)$ the set of matrices in $\mathrm{GL}_{n}(F)$ with determinant 1 . It is known that [10, Theorem, Theorems 1, and 2] if either $F$ contains at least three elements or $n>2$, then every matrix in $\mathrm{SL}_{n}(F)$ is a commutator of $\mathrm{GL}_{n}(F)$, that is, every matrix in $\mathrm{GL}_{n}(F)$ with determinant 1 has the form $B C B^{-1} C^{-1}$, in which $B$ and $C$ belong to $\mathrm{GL}_{n}(F)$. This result has motivated other decompositions of matrices in $\mathrm{SL}_{n}(F)$. For example [10], Thompson showed that if $n-2$ is not divisible by 4, then every matrix in $\mathrm{SL}_{n}(F)$ is a commutator of matrices of determinant 1. In [3], Hahn considered the decomposition of certain matrices into products of commutators of symmetric matrices [3]. B. Zheng and H. You worked on the product of commutators of symplectic transvections [12]. Recently, X. Hou considered commutators of unipotent matrices of index $2[5]$.

A matrix $A \in \mathrm{GL}_{n}(F)$ is an involution if $A$ is the inverse of itself, that is, $A^{2}=\mathrm{I}_{n}$. Here $\mathrm{I}_{n}$ is the identity matrix of $\mathrm{GL}_{n}(F)$. B. Zheng proved in [13] that if $F=\mathbb{R}$, the field of real numbers, or $F=\mathbb{C}$, the field of complex numbers, then every matrix in $\mathrm{SL}_{n}(F)$ is a product of at most two commutators of involutions in $\mathrm{GL}_{n}(F)$. This result later was extended to an arbitrary field $F$ of characteristic different from $2[6$, Theorem 2.8].

The main goal of this paper is to consider the case in which $F$ has characteristic 2. We prove the following theorem.

\footnotetext{
${ }^{*}$ Received by the editors on December 13, 2021. Accepted for publication on January 31, 2022. Handling Editor: Roger Horn. Corresponding Author: Tran Nam Son

${ }^{\dagger}$ Faculty of Mathematics and Computer Science University of Science, Ho Chi Minh City, Vietnam (trannamson1999@gmail.com).

‡Vietnam National University, Ho Chi Minh City, Vietnam

§Dong Nai University, 4 Le Qui Don Str., Tan Hiep Ward, Bien Hoa City, Dong Nai Province, Vietnam (thdung@dnpu.edu.vn, dungth0406@gmail.com).

IUniversity of Transport and Communications, Campus in Ho Chi Minh City, Vietnam (hantt_ph@utc.edu.vn, mbbien@hcmus.edu.vn).
} 
THEOREM 1. Let $F$ be a field containing at least three elements and let $n$ be a natural number greater than 1. Then every matrix in $\mathrm{SL}_{n}(F)$ is a product of at most two commutators of involutions.

We next consider special cases in which $F$ is the field $\mathbb{F}_{2}$ of two elements. We begin with $\mathrm{GL}_{2}\left(\mathbb{F}_{2}\right)$. By the fact that the determinant of every matrix in $\mathrm{GL}_{2}\left(\mathbb{F}_{2}\right)$ is 1 , one has that $\mathrm{SL}_{2}\left(\mathbb{F}_{2}\right)=\mathrm{GL}_{2}\left(\mathbb{F}_{2}\right)$. However, we show the following:

Proposition 2. Every matrix in the commutator subgroup of $\mathrm{GL}_{2}\left(\mathbb{F}_{2}\right)$ is a commutator of involutions in $\mathrm{GL}_{2}\left(\mathbb{F}_{2}\right)$.

If $n>2$, one can use [8, Lemma 1.2] to show that $A \in \mathrm{SL}_{n}\left(\mathbb{F}_{2}\right)$ is a product of at most $\frac{(n-1)(3 n+2)}{2}$ commutators of involutions of $\mathrm{GL}_{n}\left(\mathbb{F}_{2}\right)$. If $n$ is odd, we show the following result.

Proposition 3. If $n$ is an odd number greater than 1 , then every matrix of $\mathrm{SL}_{n}\left(\mathbb{F}_{2}\right)$ is a product of at most three commutators of involutions of $\mathrm{GL}_{n}\left(\mathbb{F}_{2}\right)$.

2. Proofs. To prove the main results, we need a sequence of lemmas. Let $F$ be a field and let $n>1$ be a natural number. Assume that $f(x)=x^{n}+a_{n-1} x^{n-1}+\cdots+a_{0} \in F[x]$ is a monic polynomial in one variable $x$ with coefficients $a_{i}$ in $F$. A companion matrix $C(f)$ of $f(x)$ is

$$
C(f)=\left(\begin{array}{cccc}
0 & & & -a_{0} \\
1 & & & -a_{1} \\
& \ddots & & \vdots \\
0 & & 1 & -a_{n-1}
\end{array}\right) \in \mathrm{M}_{n}(F) .
$$

The matrix $C(f)$ is invertible if and only if the constant term $a_{0}$ of $f$ is nonzero.

Let $A_{1} \in \mathrm{M}_{n_{1}}(F), A_{2} \in \mathrm{M}_{n_{2}}(F), \ldots, A_{k} \in \mathrm{M}_{n_{k}}(F)$ be square matrices of degree $n_{1}, n_{2}, \ldots, n_{k}$, respectively. The block diagonal matrix:

$$
\left(\begin{array}{cccc}
A_{1} & 0 & \cdots & 0 \\
0 & A_{2} & \cdots & 0 \\
\vdots & \vdots & \ddots & \vdots \\
0 & 0 & \cdots & A_{k}
\end{array}\right) \in \mathrm{M}_{n_{1}+n_{2}+\cdots+n_{k}}(F)
$$

is the direct sum of $A_{1}, A_{2}, \ldots, A_{k}$, denoted by $A_{1} \oplus A_{2} \oplus \cdots \oplus A_{k}$. If $f:\{1,2, \ldots, k\} \rightarrow\{1,2, \ldots, k\}, i \mapsto f(i)$ is a bijection, then $A_{1} \oplus A_{2} \oplus \cdots \oplus A_{k}$ is similar to $A_{f(1)} \oplus A_{f(2)} \oplus \cdots \oplus A_{f(k)}$.

The following result shows that each square matrix is similar to a block diagonal matrix whose diagonal blocks are companion matrices.

Lemma 4 ([7, Theorem B-3.47]). Every square matrix over the field is similar to a direct sum of companion matrices.

A square matrix of degree 1 is denoted by $(a)$, so as not to confuse it with the element $a$ in $F$.

Lemma 5 ([1, Lemma 7$])$. Let $F$ be a field containing at least four elements. Assume that $C=C(f)$ is an invertible companion matrix in $\mathrm{GL}_{n}(F)$ with respect to the polynomial $f$ of degree $n$. Let $a, b \in F \backslash\{0\}$ be such that $\operatorname{det} C=a b$. Then, $C=X Y$, in which $X$ is similar to $(a) \oplus A$ and $Y$ is similar to $(b) \oplus B$. Here,

$$
A=A_{1} \oplus A_{2} \oplus \cdots \oplus A_{k}
$$


and

$$
B=B_{1} \oplus B_{2} \oplus \cdots \oplus B_{\ell}
$$

in which each of $A_{1}, A_{2}, \ldots, A_{k}, B_{1}, B_{2}, \ldots, B_{\ell}$ is one of the following forms:

(a) (1).

(b) $\left(\begin{array}{cc}g & 0 \\ 0 & g^{-1}\end{array}\right)$, in which $g \in F \backslash\{0, \pm 1\}$.

(c) $\left(\begin{array}{cc}0 & -1 \\ 1 & q\end{array}\right)$, in which $q \in F$.

In particular, $A$ is similar to $A^{-1}$ and $B$ is similar to $B^{-1}$.

We use the following version of the preceding lemma in the proof of the main result of this paper.

Corollary 6. Let $F$ be a field of characteristic 2 containing at least four elements. Each of $C \in \mathrm{SL}_{n}(F)$ is similar to a product $A B$, in which

$$
A=A_{1} \oplus A_{2} \oplus \cdots \oplus A_{k}
$$

and

$$
B=B_{1} \oplus B_{2} \oplus \cdots \oplus B_{\ell}
$$

with

$$
A_{i}, B_{i} \in\left\{\left(\begin{array}{cc}
g & 0 \\
0 & g^{-1}
\end{array}\right) \mid g \in F \backslash\{0\}\right\} \bigcup\left\{\left(\begin{array}{ll}
0 & 1 \\
1 & q
\end{array}\right) \mid q \in F\right\}
$$

for every $i \geq 2$ and

(a) if $n$ is odd, then $A_{1}=B_{1}=(1)$;

(b) if $n$ is even, then

$$
A_{1}, B_{1} \in\left\{\left(\begin{array}{cc}
g & 0 \\
0 & g^{-1}
\end{array}\right) \mid g \in F \backslash\{0\}\right\} \bigcup\left\{\left(\begin{array}{ll}
0 & 1 \\
1 & q
\end{array}\right) \mid q \in F\right\}
$$

In particular, $A$ is similar to its inverse and so is $B$.

Proof. By Lemma 4, $C$ is similar to $C_{1} \oplus C_{2} \oplus \cdots \oplus C_{k}$, in which $C_{1}=C\left(f_{1}\right), C_{2}=C\left(f_{2}\right), \ldots, C_{k}=C\left(f_{k}\right)$ are the companion matrices of $f_{1}, f_{2}, \ldots, f_{k}$, respectively. Therefore, without loss of generality, assume that $C=C_{1} \oplus C_{2} \oplus \cdots \oplus C_{k}$, in which $C_{1}, C_{2}, \ldots, C_{k}$ are companion matrices. Each $C_{i}$ is invertible for $i=1,2, \ldots, k$ so according to Lemma $5, C_{i}$ can be expressed as the product $X_{i} Y_{i}$, in which $X_{i}$ and $Y_{i}$ are similar to $\left(a_{i}\right) \oplus A_{i},\left(b_{i}\right) \oplus B_{i}$, respectively, and $A_{i}, B_{i}$ are direct sums of the matrices of the following forms:

$$
(1),\left(\begin{array}{ll}
0 & 1 \\
1 & q
\end{array}\right),\left(\begin{array}{cc}
g & 0 \\
0 & g^{-1}
\end{array}\right)
$$

with $q \in F$ and $g \in F \backslash\{0\}$. We choose $a_{1}, a_{2}, \ldots, a_{k}$ and $b_{1}, b_{2}, \ldots, b_{k}$ in the zigzag way as follows $a_{1}=\operatorname{det} C=1, b_{1}=a_{1}^{-1} \operatorname{det} C_{1}, b_{2}=b_{1}^{-1}, a_{2}=b_{2}^{-1} \operatorname{det} C_{2}, a_{3}=a_{2}^{-1}, b_{3}=a_{3}^{-1} \operatorname{det} C_{3}$, and so on. With the similarity of direct sums and by rearrangement of the matrices of the form (1) in the front, it follows that $C$ is similar to $A B$.

Lemma 7. Let $F$ be a finite field of characteristic 2. If $x \in F \backslash\{0\}$, then there exists $y \in F \backslash\{0\}$ such that $x=y^{2}$. 
Proof. Suppose that $|F|=2^{m}$ where $m$ is a positive integer. Then $F \backslash\{0\}$ is a cyclic group of degree $2^{m}-1$ with a generator $a$. Hence, $x=a^{k}$ with $1 \leq k<2^{m}-1$. If $k$ is even, then we choose $y=a^{k / 2}$. If $k$ is odd, then we take $y=a^{\frac{2^{m}-1+k}{2}}$. Both cases lead to $y^{2}=x$.

Let $F$ be a field and let $n$ be a natural number greater than 1 . Denote by $\mathbb{L T}_{n}(F)$ and $\mathbb{U T}_{n}(F)$ the sets of lower unitriangular matrices and upper unitriangular matrices, respectively. These are triangular matrices whose diagonal entries are 1.

Lemma 8. Let $F$ be a field and let $n \geq 2$ be a positive integer. If $A \in \mathrm{SL}_{n}(F)$ is a nonscalar matrix, then there exists $P \in \mathrm{GL}_{n}(F) P^{-1} A P=B C$, in which $B \in \mathbb{L T}_{n}(F), C \in \mathbb{U T}_{n}(F)$.

Proof. This is a special case of [9, Theorem 1] .

Remark 9. Let $G$ be a group. If $g=[a, b]=a b a^{-1} b^{-1}$ is a commutator of $a, b \in G$, then $h g h^{-1}=$ $\left[h a h^{-1}, h b h^{-1}\right]$ and $g^{-1}=[b, a]$. Therefore, conjugations and inverses of commutators are commutators. Moreover, if an element in $G$ is a commutator of involutions, then so are its conjugations and inverse. These facts will be used frequently in this paper.

The following result is an intermediate step of the proof of the main theorem.

Lemma 10. Let $F$ be an infinite field. Suppose that $A \in \mathrm{SL}_{n}(F)$ and $A=B C$, in which $B \in \mathbb{L} \mathbb{T}_{n}(F)$ and $C \in \mathbb{U T}_{n}(F)$, then $A$ is a product of at most two commutators of involutions.

Proof. Consider the two following cases.

Case 1. $n$ is even, that is, $n=2 k$ for some positive integer $k$. Since $F$ is infinite, we can choose elements $x_{1}, x_{2}, \ldots, x_{k} \in F \backslash\{0\}$ such that $x_{1}^{2}, x_{2}^{2}, \ldots, x_{k}^{2}, x_{1}^{-2}, x_{2}^{-2}, \ldots, x_{k}^{-2}$ are pairwise distinct. For $1 \leq i \leq k$, put $E_{i}=\left(\begin{array}{cc}x_{i}^{2} & 0 \\ 0 & x_{i}^{-2}\end{array}\right)$ and $E=E_{1} \oplus E_{2} \oplus \cdots \oplus E_{k}$. By putting $B_{1}=B E^{-1}$ and $C_{1}=E C$, we get $A=B C=B_{1} C_{1}$, in which $B_{1}$ and $C_{1}$ have the forms:

$$
B_{1}=\left(\begin{array}{ccccc}
x_{1}^{-2} & 0 & \cdots & 0 & 0 \\
* & x_{1}^{2} & \cdots & 0 & 0 \\
\vdots & \vdots & \ddots & \vdots & \vdots \\
* & * & \cdots & x_{k}^{-2} & 0 \\
* & * & \cdots & * & x_{k}^{2}
\end{array}\right),
$$

and

$$
C_{1}=\left(\begin{array}{ccccc}
x_{1}^{2} & * & \ldots & * & * \\
0 & x_{1}^{-2} & \cdots & * & * \\
\vdots & \vdots & \ddots & \vdots & \vdots \\
0 & 0 & \cdots & x_{k}^{2} & * \\
0 & 0 & \cdots & 0 & x_{k}^{-2}
\end{array}\right) .
$$

It is known [7, Exercise B-3.32] that $C_{1}$ is similar to $E$. For $i=1, \ldots, k$, one can check that $E_{i}=\left[G_{i}, H_{i}\right]$ where $H_{i}=\left(\begin{array}{ll}0 & 1 \\ 1 & 0\end{array}\right)$ and $G_{i}=\left(\begin{array}{cc}0 & x_{i} \\ x_{i}^{-1} & 0\end{array}\right)$ are involutions in $\mathrm{GL}_{2}(F)$, so if $G=G_{1} \oplus G_{2} \oplus \cdots \oplus G_{n}$ and $H=H_{1} \oplus H_{2} \oplus \cdots \oplus H_{n}$, then $G$ and $H$ are involutions and $E=[G, H]$. By Remark 9, $C_{1}$ is a commutator of involutions. Similarly, $B_{1}$ is similar to $E^{-1}=[H, G]$. This implies that $B_{1}$ is a commutator of involutions. Thus, $A=B_{1} C_{1}$ is a product of at most two commutators of involutions. 
Case 2. $n$ is odd, that is, $n=2 k+1$ for some positive integer $k$. By the same argument as in Case 1 , we can choose $x_{1}, x_{2}, \ldots, x_{k} \in F \backslash\{0,1\}$ such that $x_{1}^{2}, x_{2}^{2}, \ldots, x_{k}^{2}, x_{1}^{-2}, x_{2}^{-2}, \ldots, x_{k}^{-2}$ are pairwise distinct. Choose $E$ as in Case 1. Then put $E^{\prime}=E \oplus(1), B_{1}^{\prime}=B E^{\prime}$ and $C_{1}^{\prime}=E^{\prime-1} C$. Using the same arguments as in Case 1 , we find that $C_{1}^{\prime}$ and $B_{1}^{\prime}$ are similar to $E^{\prime}$ and $E^{\prime-1}$, respectively. Let $G$ and $H$ be as in Case 1 . If $G^{\prime}=G \oplus(1)$ and $H^{\prime}=H \oplus(1)$, then $G^{\prime}, H^{\prime}$ are involutions and $E^{\prime}$ is the commutator of $G^{\prime}$ and $H^{\prime}$. Then, $B_{1}^{\prime}$ and $C_{1}^{\prime}$ are commutators of involutions, which implies that $A=B_{1}^{\prime} C_{1}^{\prime}$ is a product of at most two commutators of involutions.

In the proof of the main result, we use the technique of [6, Theorem 2.8]. To apply this technique, we need the following lemma.

LEMmA 11. Let $F$ be a field and let $n \geq 2$ be a positive integer. Assume that $A \in \mathrm{GL}_{n}(F)$. If $A$ is similar to $A^{-1}$, then $A^{2}$ is a commutator of involutions.

Proof. Since $A$ is similar to $A^{-1}, A=B C$, in which $B$ and $C$ are involutions [4, Theorem 1]. Hence, $A^{2}=(B C)^{2}=B C B C=B C B^{-1} C^{-1}$ is a commutator of involutions.

Now we can prove the main result of this paper.

Proof of Theorem 1. Let $C \in \mathrm{SL}_{n}(F)$. If $F$ is a field of characteristic different from 2, then $C$ is a product of at most two commutators of involutions by [6, Theorem 2.8]. Now, we suppose that the characteristic of $F$ is 2 . Consider the two following cases.

Case 1. $F$ is infinite. If $C$ is noncentral, then by Lemma 8 we have $C=A B$ with $A \in \mathbb{L}_{n}(F), B \in \mathbb{U T}_{n}(F)$. By Lemma 10, $C$ is a product of at most two commutators of involutions. Now, if $C$ is central, then there exists $\alpha \in F$ such that $C=\alpha I$. We use the arguments in the proof of [6, Theorem 2.8]. Namely, we consider the two following subcases.

Subcase 1.1. $n$ is odd. Put $\beta=\alpha^{\frac{n+1}{2}}$. Then, $\alpha=\beta^{2}$. Put $B=\operatorname{diag}\left(1, \alpha^{2}, \cdots, \alpha^{2(n-1)}\right)$. Then, $B=\operatorname{diag}\left(1, \beta^{2}, \cdots, \beta^{2(n-1)}\right)^{2}$ and $\alpha B=\operatorname{diag}\left(\beta, \beta^{3}, \cdots, \beta^{2 n-1}\right)^{2}$. Note that each of

$$
\operatorname{diag}\left(1, \beta^{2}, \cdots, \beta^{2(n-1)}\right)
$$

and

$$
\operatorname{diag}\left(\beta, \beta^{3}, \cdots, \beta^{2 n-1}\right),
$$

is similar to its inverse. Therefore, by Lemma 11, $B$ and $\alpha B$ are commutators of involutions. According to Remark $9, B^{-1}$ is also a commutator of involutions. Thus, $C=\alpha B B^{-1}$ is a product of two commutators of involutions.

Subcase 1.2. $n$ is even. Then, there exists a positive integer $m$ such that $n=2 m$. Put $B=$ $\operatorname{diag}\left(1,1, \alpha^{2}, \alpha^{2}, \cdots, \alpha^{2(m-1)}, \alpha^{2(m-1)}\right)$. For every $x \in F \backslash\{0\}$, denote $K(x)=\left(\begin{array}{ll}0 & x \\ 1 & 0\end{array}\right) . \quad$ Put $B^{\prime}=$ $K(1) \oplus K\left(\alpha^{2}\right) \oplus \cdots \oplus K\left(\alpha^{2(m-1)}\right)$ and $B^{\prime \prime}=K(\alpha) \oplus K\left(\alpha^{3}\right) \oplus \cdots \oplus K\left(\alpha^{2 m-1}\right)$. Then, $B=B^{\prime 2}$ and $\alpha B=B^{\prime \prime 2}$. By using the same arguments as in Subcase 1.1, we conclude that $B$ and $\alpha B$ are commutators of involutions. Therefore, $C=\alpha B B^{-1}$ is a product of two commutators of involutions.

Case 2. $F$ is finite. Since $F$ contains at least three elements and $F \neq \mathbb{F}_{2}, F$ has at least four elements. By Corollary 6, $C$ is similar to $A B$, in which $A$ and $B$ have the forms:

$$
A=A_{1} \oplus A_{2} \oplus \cdots \oplus A_{k}
$$


and

$$
B=B_{1} \oplus B_{2} \oplus \cdots \oplus B_{\ell}
$$

with

$$
A_{i}, B_{i} \in\left\{\left(\begin{array}{cc}
g & 0 \\
0 & g^{-1}
\end{array}\right) \mid g \in F \backslash\{0\}\right\} \bigcup\left\{\left(\begin{array}{ll}
0 & 1 \\
1 & q
\end{array}\right) \mid q \in F\right\},
$$

for every $i \geq 2$. In particular,

(a) if $n$ is odd, then $A_{1}=B_{1}=(1)$.

(b) if $n$ is even, then

$$
A_{1}, B_{1} \in\left\{\left(\begin{array}{cc}
g & 0 \\
0 & g^{-1}
\end{array}\right) \mid g \in F \backslash\{0\}\right\} \bigcup\left\{\left(\begin{array}{ll}
0 & 1 \\
1 & q
\end{array}\right) \mid q \in F\right\}
$$

By Remark 9, it suffices to assume that $C=A B$. We claim that each of the matrices $A_{1}, A_{2}, \ldots, A_{k}$, $B_{1}, B_{2}, \ldots, B_{\ell}$ is a commutator of involutions. There is nothing to prove if $n=1$. For the matrices $\operatorname{diag}\left(g, g^{-1}\right)$ with $g \in F \backslash\{0\}$, since $g \in F \backslash\{0\}$, by Lemma 7, there exists $g^{\prime} \in F \backslash\{0,1\}$ such that $g=g^{\prime 2}$ and

$$
\left(\begin{array}{cc}
g & 0 \\
0 & g^{-1}
\end{array}\right)=\left[\left(\begin{array}{cc}
0 & g^{\prime} \\
g^{\prime-1} & 0
\end{array}\right),\left(\begin{array}{ll}
0 & 1 \\
1 & 0
\end{array}\right)\right]
$$

is a commutator of involutions. For a matrix of the form $\left(\begin{array}{ll}0 & 1 \\ 1 & q\end{array}\right)$ with $q \in F$, by Lemma 7 , there exists $q^{\prime} \in F$ such that $q=q^{\prime 2}$. Then,

$$
\left(\begin{array}{ll}
0 & 1 \\
1 & q
\end{array}\right)=\left[\left(\begin{array}{cc}
q^{-1} & q^{-1}+q^{\prime} \\
q^{\prime-1} & q^{\prime-1}
\end{array}\right),\left(\begin{array}{ll}
1 & q \\
0 & 1
\end{array}\right)\right]
$$

is also a commutator of involutions. The claim is shown. Thus, each of $A$ and $B$ is a commutator of involutions. Hence, the product $C=A B$ is a product of at most two commutators of involutions.

Proof of Proposition 2. By calculating directly, we get the commutator subgroup of $\mathrm{GL}_{2}\left(\mathbb{F}_{2}\right)$ is

$$
\left[\mathrm{GL}_{2}\left(\mathbb{F}_{2}\right), \mathrm{GL}_{2}\left(\mathbb{F}_{2}\right)\right]=\left\{\left(\begin{array}{ll}
1 & 0 \\
0 & 1
\end{array}\right),\left(\begin{array}{ll}
0 & 1 \\
1 & 1
\end{array}\right),\left(\begin{array}{ll}
1 & 1 \\
1 & 0
\end{array}\right)\right\}
$$

Moreover, we have

$$
\left(\begin{array}{ll}
1 & 0 \\
0 & 1
\end{array}\right)=\left[\left(\begin{array}{ll}
0 & 1 \\
1 & 0
\end{array}\right),\left(\begin{array}{ll}
0 & 1 \\
1 & 0
\end{array}\right)\right],\left(\begin{array}{ll}
1 & 1 \\
1 & 0
\end{array}\right)=\left[\left(\begin{array}{ll}
1 & 1 \\
0 & 1
\end{array}\right),\left(\begin{array}{ll}
1 & 0 \\
1 & 1
\end{array}\right)\right]
$$

and

$$
\left(\begin{array}{ll}
0 & 1 \\
1 & 1
\end{array}\right)=\left[\left(\begin{array}{ll}
1 & 0 \\
1 & 1
\end{array}\right),\left(\begin{array}{ll}
1 & 1 \\
0 & 1
\end{array}\right)\right]
$$

By Remark 9, the proof is complete. 
Electronic Journal of Linear Algebra, ISSN 1081-3810

A publication of the International Linear Algebra Society

Volume 38, pp. 123-130, February 2022.

A matrix $A \in \mathrm{GL}_{n}(F)$ of degree $n$ over a field $F$ is a transvection if the rank of $A-\mathrm{I}_{n}$ is 1 and $\left(A-\mathrm{I}_{n}\right)^{2}=0$. For example, a matrix of the form $\left(\begin{array}{ccccc}1 & & & & * \\ & 1 & & & * \\ & 1 & & * \\ & & \ddots & & \vdots \\ & & & 1 & * \\ & & & & \\ & & & \end{array}\right)$ is a transvection. It is known that all transvections are similar [2, Proposition 1.5].

Proof of Proposition 3. Let $A \in \mathrm{SL}_{n}\left(\mathbb{F}_{2}\right)$. By [11, Theorem 2], we can choose $B$ and $C \in \mathrm{GL}_{n}\left(\mathbb{F}_{2}\right)$ such that $A=B C$, in which $B$ and $C$ are, respectively, similar to

$$
B_{1}=\left(\begin{array}{cccccc}
0 & & & & & 1 \\
1 & 0 & & & & \\
& 1 & \ddots & & & \\
& & \ddots & 0 & & \\
& & & 1 & 0 & \\
& & & & 1 & 0
\end{array}\right) \text { and } C_{1}=\left(\begin{array}{cccccc}
0 & & & & & 1 \\
1 & 0 & & & & * \\
& 1 & \ddots & & & \vdots \\
& & \ddots & 0 & & * \\
& & & 1 & 0 & * \\
& & & & 1 & *
\end{array}\right) .
$$

Observe that the minimal polynomial of $B_{1}$ is $t^{n}-1$, so $B_{1}^{n+1}=B_{1}$. Since $n+1$ is even, put $B_{2}=B_{1}^{\frac{n+1}{2}}$. Then $B_{1}=B_{2}^{2}$ and

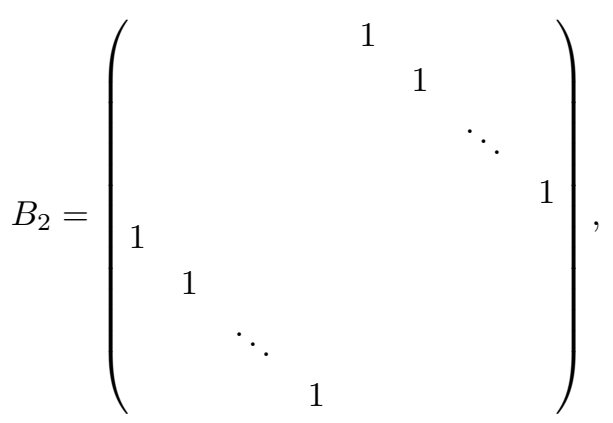

which is the product

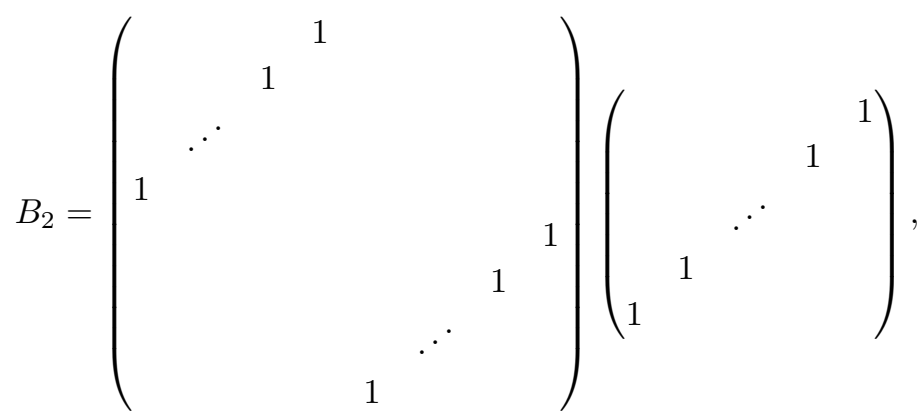


of involutions. Hence, $B_{1}=B_{2}^{2}$ is a commutator of involutions. Now put $C_{2}=B_{1}^{-1} C_{1}$. Then,

$$
C_{2}=\left(\begin{array}{cccccc}
1 & & & & & * \\
& 1 & & & & * \\
& & 1 & & & * \\
& & & \ddots & & \vdots \\
& & & & 1 & * \\
& & & & & 1
\end{array}\right) .
$$

If $C_{2}=\mathrm{I}_{n}$, then $C_{2}$ is a commutator of involutions, so we assume that $C_{2} \neq \mathrm{I}_{n}$. Then, $C_{2}$ is a transvection, which implies that $C_{2}$ is similar to $\left(\begin{array}{ll}1 & 1 \\ 0 & 1\end{array}\right) \oplus \mathrm{I}_{n-2}$, as $\left(\begin{array}{ll}1 & 1 \\ 0 & 1\end{array}\right) \oplus \mathrm{I}_{n-2}$ is also a transvection. Observe that

$$
\left(\begin{array}{lll}
1 & 1 & 0 \\
0 & 1 & 0 \\
0 & 0 & 1
\end{array}\right) \oplus \mathrm{I}_{n-3}=\left[\left(\begin{array}{lll}
1 & 0 & 1 \\
0 & 1 & 0 \\
0 & 0 & 1
\end{array}\right) \oplus \mathrm{I}_{n-3},\left(\begin{array}{lll}
1 & 0 & 0 \\
0 & 1 & 0 \\
0 & 1 & 1
\end{array}\right) \oplus \mathrm{I}_{n-3}\right],
$$

is a commutator of involutions, so $C_{1}=B_{1} C_{2}$ is a product of two commutators of involutions. Thus, $A$ is a product of at most three commutators of involutions.

Acknowledgments. The authors would like to express their sincere gratitude to the referee for his/her comments and suggestions due to which the paper was greatly improved. The fourth author is funded by Vietnam National University HoChiMinh City (VNUHCM) under grant number T2022-18-03.

\section{REFERENCES}

[1] J.D. Botha. On a unification result by A.R. Sourour concerning commutators and products of involutions. Linear Algebra Appl., 416:872-879, 2006.

[2] L.C. Grove. Classical Groups and Geometric Algebra. Graduate Studies in Mathematics. Vol. 39, x-169. American Mathematical Society, Providence, RI, 2002.

[3] A.J. Hahn. The elements of the orthogonal group $\Omega_{n}(V)$ as products of commutators of symmetries. J. Algebra, 184:927$944,1996$.

[4] F. Hoffman and E. Paige. Products of two involutions in the general linear group. Math. J., Indiana Univ., 20:1017-1020, 1971.

[5] X. Hou. Decomposition of matrices into commutators of unipotent matrices of index 2. Electron. J. Linear Algebra, $37: 31-34,2021$

[6] X. Hou. Decomposition of infinite matrices into products of commutators of involutions. Linear Algebra Appl., 563:231239, 2019

[7] J.J. Rotman. Advanced Modern Algebra: Third Edition, Part 1. American Mathematical Society, Providence, RI, 2015. xiv+706 pp. ISBN: 978-1-4704-1554-9.

[8] A.S. Sivatski and A.V. Stepanov. On the word length of commutators in $\mathrm{GL}_{n}(R)$. K-Theory, 17:295-302, 1999.

[9] A.R. Sourour. A factorization theorem for matrices. Linear Multilinear Algebra, 19:141-147, 1986.

[10] R.C. Thompson. Commutators in the special and general linear groups. Trans. Amer. Math. Soc., 101:16-33, 1961.

[11] L.N. Vaserstein and E. Wheland. Commutators and companion matrices over rings of stable rank 1. Linear Algebra Appl., 142:263-277, 1990.

[12] B. Zheng and H. You. Products of commutators of symplectic transvections. Chinese Ann. Math., 20A:277-282, 1999.

[13] B. Zheng. Decomposition of matrices into commutators of involutions. Linear Algebra Appl., 347:1-7, 2002. 